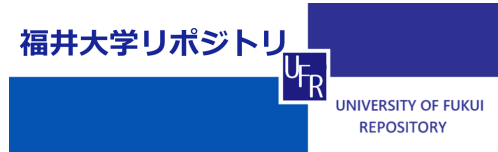

Meani ng of anti paral I el proton and neutron angul ar noment $a$ at I ow spi ns

\begin{tabular}{|l|l|}
\hline 著者 & TAJI MA Naoki , OTSUKA Takahar u \\
\hline $\begin{array}{l}\text { j our nal or } \\
\text { publ i cat i on t i t l e }\end{array}$ & PHYSI CAL REVI EW C \\
\hline vol une & 84 \\
\hline page $r$ ange & 064316-1-064316- 8 \\
\hline year & 2011- 01 \\
\hline URL & ht t p: //hdl . handl e. net /10098/4849 \\
\hline
\end{tabular}




\title{
Meaning of antiparallel proton and neutron angular momenta at low spins
}

\author{
Naoki Tajima ${ }^{1}$ and Takaharu Otsuka ${ }^{2,3}$ \\ ${ }^{1}$ Department of Applied Physics, University of Fukui, 3-9-1 Bunkyo, Fukui 910-8507, Japan \\ ${ }^{2}$ Department of Physics and Center for Nuclear Study, University of Tokyo, Hongo, Bunkyo-ku, Tokyo, 113, Japan \\ ${ }^{3}$ National Superconducting Cyclotron Laboratory, Michigan State University, East Lansing, Michigan 48824, USA \\ (Received 24 July 2011; revised manuscript received 23 November 2011; published 15 December 2011)
}

\begin{abstract}
The meaning of the fact that the angular momenta of protons and neutrons are oriented in opposite directions, shown by Otsuka [Phys. Rev. Lett. 71, 1804 (1993)] for the angular-momentum-projected Nilsson wave functions, is reexamined using a two-rotor model. It is shown that this fact does not necessarily mean an unphysical situation that proton and neutron ellipsoids are rotating freely in opposite directions. On the contrary, it originates in a close binding of the two ellipsoids, which is accompanied by a large spreading of the relative angular momentum due to the uncertainty principle. It is also demonstrated that the elimination of the spurious center-of-mass motion does not substantially change the situation.
\end{abstract}

DOI: 10.1103/PhysRevC.84.064316

PACS number(s): 21.60.Ev, 21.10.Re

\section{INTRODUCTION}

Rotational motion is one of the most basic collective modes in nuclei, and well-deformed nuclei exhibit the ideal pattern almost perfectly. This motion has been described as the rotation of an ellipsoid [1]. Because the nucleus consists of protons and neutrons, it is of interest to study the structure of the rotational states in terms of proton-neutron degrees of freedom.

In this paper we revisit the analysis of the correlation between the motion of a proton ellipsoid and that of a neutron ellipsoid presented for the ground-state rotational band in Refs. [2-4]. In fact, it was shown in Refs. [2-4] that the total angular momentum of a proton ellipsoid, $\boldsymbol{J}_{\mathrm{p}}$, and that of a neutron ellipsoid, $\boldsymbol{J}_{\mathrm{n}}$, are correlated in completely (nearly) opposite directions in the ground (low-spin) state of deformed nuclei. The total angular momentum is defined as $\boldsymbol{J}=\boldsymbol{J}_{\mathrm{p}}+\boldsymbol{J}_{\mathrm{n}}$ from $\boldsymbol{J}_{\mathrm{p}}$ and $\boldsymbol{J}_{\mathrm{n}}$, with their magnitudes $J, J_{\mathrm{p}}$, and $J_{\mathrm{n}}$, respectively. The aforementioned correlation means that for the states with $J=0$ (low $J$ values), $\boldsymbol{J}_{\mathrm{p}}$ and $\boldsymbol{J}_{\mathrm{n}}$ are pointing exactly (nearly) in opposite directions [2].

Regarding the relative motion between the proton and neutron ellipsoids, the scissors mode has been known as an excitation on top of the ground state of deformed nuclei, apart from the rotational excitation. The scissors mode is viewed as an isovector-type oscillation of the relative angle between these ellipsoids. The scissors mode was predicted, for instance, in Ref. [5] and observed for the first time in Ref. [6] for ${ }^{156} \mathrm{Gd}$. Since then, scissors-type $M 1$ excitations have been observed in many nuclei, including the most recent one in ${ }^{232} \mathrm{Th}$ [7]. The photon scattering facilities both at the S-DALINAC and HIGS at Duke University have produced and are still producing a wealth of data on the scissors mode and related phenomena. One finds many past and on-going developments in a review [8], as well as recent theoretical works (for example, Refs. $[9,10])$. The scissors mode can be of high interest in neutron-rich exotic nuclei as discussed later in this section. Furthermore, the importance of the scissors mode is underlined by the fact that it is also found in other many-body systems such as trapped Bose-Einstein condensates [8]. A consistent picture of the scissors mode and the above-mentioned $J_{\mathrm{p}^{-}} J_{\mathrm{n}}$ correlation will be shown in some detail below.
The classical-mechanical picture of the rotational band is very different from the one with the opposite $J_{\mathrm{p}}-J_{\mathrm{n}}$ correlation. Namely, the lowest-energy state for a given total angular momentum (yrast state) is constructed by simply dividing $J$ into $J_{\mathrm{p}}$ and $J_{\mathrm{n}}$ in the ratio of the moments of inertia. In such states, the angular momentum vector of protons and that of neutrons are parallel, and $J_{\mathrm{p}}=J_{\mathrm{n}}=0$ holds for the $J=0$ ground state.

In quantum mechanics, however, one has to consider large magnitudes and fluctuations of $\boldsymbol{J}_{\mathrm{p}}$ and $\boldsymbol{J}_{\mathrm{n}}$ caused by deformed shapes of the ellipsoids which are consequences of self-consistent deformed mean fields. To be more precise, when a state with a good $J$ value is expressed in the laboratory frame by a superposition of components of good $J_{\mathrm{p}}$ and $J_{\mathrm{n}}$ values, components with various large values of $J_{\mathrm{p}}$ and $J_{\mathrm{n}}$ are included significantly even in the states of $J \sim 0$. In such states, $\boldsymbol{J}_{\mathrm{p}}$ and $\boldsymbol{J}_{\mathrm{n}}$ are coupled in (almost) opposite directions due to the requirement that their vector sum is of small magnitude. This situation is shown schematically in Fig. 1. Figure 1(a) indicates how the rotation of the proton ellipsoid is coupled with the rotation of the neutron ellipsoid in the $J=0$ ground state. The angular momentum vectors $J_{\mathrm{p}}$ and $\boldsymbol{J}_{\mathrm{n}}$ have been introduced above. The vectors shown in Fig. 1 are schematic images of a representative situation (or snapshots). On the other hand, the total angular momentum is fixed to its magnitude $J$ and $z$ component $J_{z}$ in Fig. 1, as for instance $J=J_{z}=0$ in Fig. 1(a). Figure 1(a) implies that both ellipsoids are rotating about the $y$ axis, i.e., $\boldsymbol{J}_{\mathrm{p}}$ and $\boldsymbol{J}_{\mathrm{n}}$ are on the $y$ axis, but they should also be rotated as a whole about the $z$ axis. The latter rotation is implemented already in the axially symmetric intrinsic wave function. We thus generate the $J=0$ state in the laboratory frame, keeping the present $\boldsymbol{J}_{\mathrm{p}}-\boldsymbol{J}_{\mathrm{n}}$ correlation for their relative relation. This is consistent with the usual method of angular momentum projection. We mention once more that Fig. 1 is a schematic illustration of a representative portion of the whole wave function.

The change from Figs. 1(a) to 1(b) implies a transition from the $J=0$ ground state to an excited state in the rotational band with a low $J$ value. In this state, the $\boldsymbol{J}_{\mathrm{p}}-\boldsymbol{J}_{\mathrm{n}}$ correlation is maintained to a similar extent to the one in the $J=0$ 
(b) rotation (low $J$ )



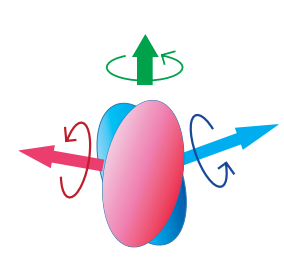



(d) scissors mode $C$ B

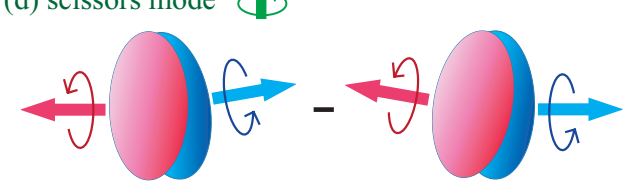

(c) rotation (high $J$ )

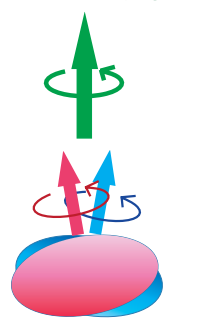

FIG. 1. (Color online) Schematic illustration of angular momentum coupling between proton (left, red) and neutron (right, blue) ellipsoids, for the ground state, rotational excitation, and scissors-mode excitation. The angular momenta of proton and neutron ellipsoids are shown by arrows $\boldsymbol{J}_{\mathrm{p}}$ and $\boldsymbol{J}_{\mathrm{n}}$, respectively. The short arrows (in green) in (b) and (c) represent the rotational angular momentum $\boldsymbol{J}$, while the one in (d) indicates the scissors-mode $K$ quantum number. Thin vectors in (b) indicate components of $\boldsymbol{J}_{\mathrm{p}}$ and $\boldsymbol{J}_{\mathrm{n}}$, perpendicular to $\boldsymbol{J}$. The angular momentum vector patterns shown here indicate schematically representative components of actual wave functions.

state, while the low $J$ value is generated mainly by tilting the two axial vectors, resulting in the rotational excitation [2]. In Ref. [2], the lengths $J_{\mathrm{p}}$ and $J_{\mathrm{n}}$, and the angle between $\boldsymbol{J}_{\mathrm{p}}$ and $\boldsymbol{J}_{\mathrm{n}}$ are depicted using mean values obtained, for the state of a given $J$, from expectation values of operators $\left(\hat{\boldsymbol{J}}_{\mathrm{p}} \cdot \hat{\boldsymbol{J}}_{\mathrm{p}}\right),\left(\hat{\boldsymbol{J}}_{\mathrm{n}} \cdot \hat{\boldsymbol{J}}_{\mathrm{n}}\right)$, and $\left(\hat{\boldsymbol{J}}_{\mathrm{p}} \cdot \hat{\boldsymbol{J}}_{\mathrm{n}}\right)$, where $(\cdot)$ means a scalar product. Figure 1 is nothing but a schematic illustration of these quantities. The right part of Fig. 1(b) exhibits how the coupling of $\boldsymbol{J}_{\mathrm{p}}$ and $\boldsymbol{J}_{\mathrm{n}}$ is made: the vector sum becomes $\boldsymbol{J}$, and we put it, for the sake of simplicity, so that $J_{z}=J$. (Note that the direction of $\boldsymbol{J}$ can be easily changed by making the lengths of the two vectors slightly different.) There are components perpendicular to this summed vector, as shown by thin horizontally placed vectors in the right part of Fig. 1(b). They represent the present $\boldsymbol{J}_{\mathrm{p}}-\boldsymbol{J}_{\mathrm{n}}$ correlation for this particular state. The amount of this correlation changes as a function of $J$.

The rotational excitation is thus made by coherent changes of $\boldsymbol{J}_{\mathrm{p}}$ and $\boldsymbol{J}_{\mathrm{n}}$, on top of the $\boldsymbol{J}_{\mathrm{p}^{-}} \boldsymbol{J}_{\mathrm{n}}$ correlation in the ground state. The changes are partly due to the tilting of these vectors toward the positive direction of the $z$ axis and partly due to the changes of the magnitudes, $J_{\mathrm{p}}$ and $J_{\mathrm{n}}$. Due to these changes, the present $\boldsymbol{J}_{\mathrm{p}}-\boldsymbol{J}_{\mathrm{n}}$ correlation becomes weaker in general as compared to $J$. This reduction of the $\boldsymbol{J}_{\mathrm{p}}-\boldsymbol{J}_{\mathrm{n}}$ correlation for higher $J$ values has been confirmed for examples shown in Ref. [2]. An illustration for a high $J$ value is shown in Fig. 1(c) schematically. This is closer to the classical image of the rotation. We point out that both rotational excitation and reduction of the $\boldsymbol{J}_{\mathrm{p}^{-}} \boldsymbol{J}_{\mathrm{n}}$ correlation occur due to the changes of $\boldsymbol{J}_{\mathrm{p}}$ and $\boldsymbol{J}_{\mathrm{n}}$ as $\boldsymbol{J}$ increases, while they are always perpendicular to the symmetry axes in the intrinsic frame.

When the present $\boldsymbol{J}_{\mathrm{p}}-\boldsymbol{J}_{\mathrm{n}}$ correlation was pointed out in Ref. [2], it was never described as "a free motion." Nevertheless, in some cases mentioned below, this correlation seems to have been misinterpreted as signifying the disappearance of the strong correlation between the two ellipsoids.
In Ref. [11], it was argued that such a picture (referred to as "contra-rotation") was unlikely from physical points of view and the author of Ref. [11] tried to show that the proton and the neutron angular momenta would point to the same direction if they were defined in the center of mass (c.m.) frame rather than in the laboratory frame as in Ref. [2]. Since then, two papers [12,13] have cited Ref. [11] as a paper solving the controversy. In this paper, we first show that such an argument is applicable only to two-particle (spinless) systems but not to deformed nuclei, which are composed of many nucleons. Moreover, in the latter case our classical estimation indicates that it is not essential whether one defines the angular momenta in the laboratory frame or in the c.m. frame.

Next, we present insight, by taking a two-rotor system in two-dimensional space, into the question regarding the antiparallel coupling between the angular momentum of the proton rotor and that of neutron one, in states of low total angular momenta. It will be demonstrated that the present antiparallel-coupling feature does not necessarily indicate a free "contra-rotation" but is more like an indispensable correlation so as to produce the tight mutual binding of the proton and neutron ellipsoids in rotational bands.

We now discuss how the present $\boldsymbol{J}_{\mathrm{p}}-\boldsymbol{J}_{\mathrm{n}}$ correlation can be related to the scissors mode. One can conceive another excitation mode as depicted in Fig. 1(d) schematically. Note that there is a minus sign in Fig. 1(d). This corresponds to the scissors mode. The scissors excitation can be modeled by the transition operator $\hat{\boldsymbol{J}}_{\mathrm{p}}-\hat{\boldsymbol{J}}_{\mathrm{n}}$. The minus sign in Fig. 1(d) is due to the minus sign in this operator. The additive operator $\hat{\boldsymbol{J}}_{\mathrm{p}}+\hat{\boldsymbol{J}}_{\mathrm{n}}$ is the total angular momentum, yielding no transition.

The summed $B(M 1)$ value to the scissors mode is given by the expectation value of a scalar product, $\left(\hat{\boldsymbol{J}}_{\mathrm{p}}-\hat{\boldsymbol{J}}_{\mathrm{n}}\right)^{2}$. This operator is decomposed as $\hat{\boldsymbol{J}}_{\mathrm{p}}^{2}+\hat{\boldsymbol{J}}_{\mathrm{n}}^{2}-2\left(\hat{\boldsymbol{J}}_{\mathrm{p}} \cdot \hat{\boldsymbol{J}}_{\mathrm{n}}\right)$. The antiparallel coupling of $\boldsymbol{J}_{\mathrm{p}}$ and $\boldsymbol{J}_{\mathrm{n}}$ in the ground state [Fig. 1(a)] produces a large negative expectation value of 
$\left(\hat{\boldsymbol{J}}_{\mathrm{p}} \cdot \hat{\boldsymbol{J}}_{\mathrm{n}}\right)$, giving rise to a large $B(M 1)$. Thus, the antiparallel $\boldsymbol{J}_{\mathrm{p}}-\boldsymbol{J}_{\mathrm{n}}$ correlation can be viewed as the zero-point oscillation, or the ground-state correlation, of the scissors mode, enhancing its strength. The $B(M 1)$ strength to the scissors mode has in fact been analyzed in Ref. [4] in connection to the present $\boldsymbol{J}_{\mathrm{p}}-\boldsymbol{J}_{\mathrm{n}}$ correlation, and the resultant $B(M 1)$ value $\left(\sim 4\left(\mu_{N}^{2}\right)\right.$ for $\left.{ }^{156} \mathrm{Sm}[4]\right)$ is in a reasonable agreement with the systematic behavior of the observed $B(M 1)$ sum rule $[8,14]$.

What we want to stress is that rotational states and the scissors mode are closely related to this antiparallel $\boldsymbol{J}_{\mathrm{p}}-\boldsymbol{J}_{\mathrm{n}}$ correlation. Rotational states are built on this correlation. The $B(M 1)$ value of the scissors mode vanishes if this correlation vanishes. In general, the scissors-like oscillation arises on top of the antiparallel correlation of the angular momenta of partial systems. In this sense, it is very important to settle the present issue and fill a pitfall in the road to future research and generalizations of the scissors mode.

Among future perspectives of studies on the scissors mode, a possibility to make use of measured magnetic dipole strengths as a new indicator of sudden shape phase transitions is mentioned in Ref. [8]. This idea is based on the proportionality of the summed $M 1$ strength to the square of the nuclear deformation parameter [15]. A deeper understanding of the present issue may provide us with a way to understand this proportionality. Namely, the summed $M 1$ strength is proportional to the expectation value of $\left(\hat{\boldsymbol{J}}_{\mathrm{p}}-\hat{\boldsymbol{J}}_{\mathrm{n}}\right)^{2}$ with respect to the ground state [4], which, in turn, includes a term proportional to the mean square value of the angle between the axes of proton and neutron ellipsoids. For stronger deformation, the ellipsoids become thinner, and an attractive interaction between the two ellipsoids makes this angle smaller because an increase of the angle results in a decrease of the overlap, i.e., the attraction. Thus, one is led to a larger $B(M 1)$ value for a stronger deformation.

One of the current interests in nuclear physics is the scissors mode in neutron-rich exotic nuclei. The neutron skin may change the shape of the ellipsoid and also the correlation between the proton and neutron ellipsoids. This can affect both the rotational properties and the scissors mode, but the latter can be more sensitive because the strength of the mode is directly linked to the proton-neutron difference of the angular momentum contents. In this sense, a comprehensive understanding of the rotation and scissors mode is very important for future nuclear physics [8].

Further clarification of the antiparallel coupling of proton and neutron rotations and its consequence in the $M 1$ excitation therefore seems to be of vital and wide interest.

\section{EFFECTS OF REMOVAL OF THE CENTER-OF-MASS MOTION}

Let us consider a system of $N(\geqslant 2)$ particles, composed of $N_{\mathrm{p}}(\geqslant 1)$ protons and $N_{\mathrm{n}}\left(=N-N_{\mathrm{p}} \geqslant 1\right)$ neutrons. The position and linear momentum of the $i$ th $(1 \leqslant i \leqslant N)$ particle are denoted as $\boldsymbol{r}_{i}$ and $\boldsymbol{p}_{i}$, respectively. For the sake of simplicity we regard the mass of a proton and that of a neutron as equal and we do not consider the intrinsic spin. These simplifications will not alter the conclusion of this section.
The total angular momentum of the $N$ particles defined in the laboratory frame is denoted as

$$
\boldsymbol{l}=\sum_{i} \boldsymbol{r}_{i} \times \boldsymbol{p}_{i}
$$

while that in the c.m. frame is

$$
\tilde{\boldsymbol{l}}=\sum_{i} \tilde{\boldsymbol{r}}_{i} \times \tilde{\boldsymbol{p}}_{i}
$$

where

$$
\tilde{\boldsymbol{r}}_{i}=\boldsymbol{r}_{i}-\frac{1}{N} \sum_{j} \boldsymbol{r}_{j}
$$

and

$$
\tilde{\boldsymbol{p}}_{i}=\boldsymbol{p}_{i}-\frac{1}{N} \sum_{j} \boldsymbol{p}_{j} .
$$

The summation over $i$ or $j$ in Eqs. (1)-(4) should be taken over $1 \leqslant i \leqslant N$ or $1 \leqslant j \leqslant N$. In this section, as in Ref. [11], vectors with a tilde over them mean those defined in the c.m. frame of the total system. The total angular momentum can be divided into the proton and the neutron contributions,

$$
\boldsymbol{l}=\boldsymbol{l}_{\mathrm{p}}+\boldsymbol{l}_{\mathrm{n}}, \quad \tilde{\boldsymbol{l}}=\tilde{\boldsymbol{l}}_{\mathrm{p}}+\tilde{\boldsymbol{l}}_{\mathrm{n}} .
$$

In this paper, the subscript $\mathrm{p}$ or $\mathrm{n}$ indicates, respectively, quantities related to the proton system or the neutron system and will be denoted collectively by $\tau(=\mathrm{p}, \mathrm{n})$. The quantities on the right-hand sides of Eqs. (5) are then defined by restricting the summations in Eqs. (1) and (2), $\sum_{i}=\sum_{i=1}^{N_{\mathrm{p}}+N_{\mathrm{n}}}$, to either protons, $\sum_{i \in \mathrm{p}}=\sum_{i=1}^{N_{\mathrm{p}}}$, or neutrons, $\sum_{i \in \mathrm{n}}=\sum_{i=N_{\mathrm{p}}+1}^{N_{\mathrm{p}}+N_{\mathrm{n}}}$. We here introduce the angular momentum due to the c.m. motion of the proton (or neutron) system,

$$
\boldsymbol{L}_{\tau}=\boldsymbol{R}_{\tau} \times \boldsymbol{P}_{\tau},
$$

where $\boldsymbol{R}_{\tau}$ denotes the position of the c.m. of the type- $\tau$ nucleon system given by

$$
\boldsymbol{R}_{\tau}=\frac{1}{N_{\tau}} \sum_{i \in \tau} \boldsymbol{r}_{i},
$$

and $\boldsymbol{P}_{\tau}$ means the total momentum of the system written as

$$
\boldsymbol{P}_{\tau}=\sum_{i \in \tau} \boldsymbol{p}_{i}
$$

We then express $\boldsymbol{l}_{\tau}$ as

$$
\boldsymbol{l}_{\tau}=\boldsymbol{L}_{\tau}+\boldsymbol{\Sigma}_{\tau},
$$

where $\boldsymbol{\Sigma}_{\tau}$ is nothing but the angular momentum of the type- $\tau$ nucleon system in its own c.m. frame,

$$
\boldsymbol{\Sigma}_{\tau}=\sum_{i \in \tau}\left(\boldsymbol{r}_{i}-\boldsymbol{R}_{\tau}\right) \times\left(\boldsymbol{p}_{i}-\frac{1}{N_{\tau}} \boldsymbol{P}_{\tau}\right) .
$$

Note that, if $N_{\tau}=1, \boldsymbol{\Sigma}_{\tau}=\mathbf{0}$ because $\boldsymbol{R}_{\tau}$ is equal to the position of the sole type- $\tau$ nucleon.

Likewise, the angular momentum of the c.m. motion of the type- $\tau$ nucleon system measured from the total c.m. is written as

$$
\tilde{\boldsymbol{L}}_{\tau}=\tilde{\boldsymbol{R}}_{\tau} \times \tilde{\boldsymbol{P}}_{\tau},
$$


where $\tilde{\boldsymbol{R}}_{\tau}$ and $\tilde{\boldsymbol{P}}_{\tau}$ are defined with respect to the total c.m. but are otherwise similar to Eqs. (7) and (8):

$$
\tilde{\boldsymbol{R}}_{\tau}=\frac{1}{N_{\tau}} \sum_{i \in \tau} \tilde{\boldsymbol{r}}_{i}
$$

and

$$
\tilde{\boldsymbol{P}}_{\tau}=\sum_{i \in \tau} \tilde{\boldsymbol{p}}_{i}
$$

We point out that, from $N_{\mathrm{p}} \tilde{\boldsymbol{R}}_{\mathrm{p}}+N_{\mathrm{n}} \tilde{\boldsymbol{R}}_{\mathrm{n}}=0$ and $\tilde{\boldsymbol{P}}_{\mathrm{p}}+\tilde{\boldsymbol{P}}_{\mathrm{n}}=$ 0 , an equality can be derived:

$$
N_{\mathrm{p}} \tilde{\boldsymbol{L}}_{\mathrm{p}}=N_{\mathrm{n}} \tilde{\boldsymbol{L}}_{\mathrm{n}}
$$

One can also show that $\boldsymbol{\Sigma}_{\tau}$ of Eq. (10) appears again in the relation between angular momenta in the c.m. frame,

$$
\tilde{\boldsymbol{l}}_{\tau}=\tilde{\boldsymbol{L}}_{\tau}+\boldsymbol{\Sigma}_{\tau} \text {. }
$$

This is because $\boldsymbol{\Sigma}_{\tau}$ is transformed like intrinsic spins; i.e., it is independent of the position and the velocity of the origin of the coordinates. If the system were a rigid body, it would represent the angular momentum due to the spinning of the rigid body. Indeed, in order to take into account true intrinsic spins of type- $\tau$ nucleons, one only has to add the spins to $\boldsymbol{\Sigma}_{\tau}$ while leaving $\boldsymbol{L}_{\tau}$ and $\tilde{\boldsymbol{L}}_{\tau}$ unchanged.

In terms of the quantities defined above, the argument of Ref. [11] is that, although $l_{\mathrm{p}}$ and $\boldsymbol{l}_{\mathrm{n}}$ may be antiparallel, $\tilde{l}_{\mathrm{p}}$ and $\tilde{l}_{\mathrm{n}}$ are always parallel. This argument is correct in the case of two (spinless) particles because $\boldsymbol{\Sigma}_{\tau}=0$ and Eq. (14). In systems with more than two particles, however, $\boldsymbol{\Sigma}_{\tau}$ is generally not zero. On the contrary, according to a classical estimation shown in the Appendix, $\Sigma_{\tau} \propto N_{\tau}^{1 / 2}$ is likely to be much larger than $\boldsymbol{L}_{\tau} \propto N_{\tau}^{0}$ and $\tilde{\boldsymbol{L}}_{\tau} \propto N_{\tau}^{0}$. Therefore the difference between $\boldsymbol{l}_{\tau}$ and $\tilde{\boldsymbol{l}}_{\tau}$ is much smaller than either value and both of them are almost equal to $\boldsymbol{\Sigma}_{\tau}$. It follows that, if they are antiparallel in the laboratory frame, they are also antiparallel in the c.m. frame.

We mention that $\tilde{\boldsymbol{L}}_{\mathrm{p}}=\tilde{\boldsymbol{L}}_{\mathrm{n}}=0$ holds in some shell-model calculations by setting the c.m. motions of protons and neutrons separately to the nodeless $s$ state, for the purpose of the removal of spurious motion. This can be fulfilled by taking a full $L S$ valence shell (e.g., $s d$ shell), and we can confirm the same antiparallel relation between proton and neutron angular momenta.

\section{ANALYSIS WITH A TWO-ROTOR MODEL}

Next, we discuss the implication of the opposite orientations of the proton and the neutron angular momenta using a tworotor model. One of the rotors represents all the protons while the other represents neutrons. They are constrained to rotate about a space-fixed axis. In other words, the rotors rotate in the two-dimensional space perpendicular to this axis. Thus, in this model, there are just two dynamical variables, $\varphi_{\mathrm{p}}$ and $\varphi_{\mathrm{n}}$, which are the azimuthal angles to specify the orientations of the proton and the neutron rotors, respectively. They are restricted to a domain $-\pi \leqslant \varphi_{\mathrm{p}, \mathrm{n}} \leqslant \pi$ : We do not impose " $\mathcal{R}_{1}$ symmetry" (invariance of the wave function as for a $\pi$ rotation [1]) for the sake of simplicity.
We first introduce an intrinsic wave function containing the quantum fluctuations of the orientations of the two rotors. This wave function corresponds to the Nilsson-BCS wave function used in Ref. [2]. The present intrinsic wave function is written as

$$
\psi\left(\varphi_{\mathrm{p}}, \varphi_{\mathrm{n}}\right)=f_{\mathrm{p}}\left(\varphi_{\mathrm{p}}\right) f_{\mathrm{n}}\left(\varphi_{\mathrm{n}}\right),
$$

where $f_{\mathrm{p}}\left(f_{\mathrm{n}}\right)$ is the proton (neutron) intrinsic wave function. As the effect of the restoration of the rotational symmetry is the issue, this simple wave function is sufficient for the following discussion. We choose $f_{\mathrm{p}}=f_{\mathrm{n}}=f$ for simplicity. To make them localized around $\varphi=0$, we assume the following form:

$$
f(\varphi)=\sum_{m=-\infty}^{\infty} f_{m} e^{i m \varphi}, \quad f_{m}=\mathcal{N}_{\nu} e^{-|a m|^{\nu}},
$$

where $v$ is a parameter taking on positive real values. $\mathcal{N}_{v}$ is a normalization factor such that

$$
\int_{-\pi}^{\pi}|f(\varphi)|^{2} d \varphi=1
$$

or, equivalently,

$$
\sum_{m=-\infty}^{\infty}\left|f_{m}\right|^{2}=\frac{1}{2 \pi}
$$

With larger values of $v$, one can reduce more sharply contributions from components with higher angular momenta in $f(\varphi)$. For $v=2, f_{m}$ is a Gaussian function with $\left\langle m^{2}\right\rangle=(1 / 2 a)^{2}$. We set $a=0.1$ throughout this paper. When $a$ is much smaller than $\pi, f(\varphi)$, too, is to a good approximation a Gaussian function with $\left\langle\varphi^{2}\right\rangle=a^{2}$. In similar approximations, one finds $f(\varphi) \propto$ $\left(\varphi^{2}+a^{2}\right)^{-1}$ for $\nu=1$ and $f(\varphi) \propto \sin (\varphi / a) / \varphi$ for $\nu \rightarrow \infty$. We regard $v=2$ as the most reasonable value because the distribution of $m$ coincides with that of the thermodynamic equilibrium $\exp \left(-\epsilon_{m} / k T\right)$, where $\epsilon_{m} \propto m^{2}$. Later, we also consider a case of a finite number of angular momentum components by restricting the summation in Eq. (17) to $-15 \leqslant$ $m \leqslant 15$ for $v=2$.

We introduce an operator $\hat{P}_{M}$ which projects $\psi\left(\varphi_{\mathrm{p}}, \varphi_{\mathrm{n}}\right)$ onto the eigenspace belonging to the eigenvalue $M$ of the total (two-dimensional) angular momentum

$$
\hat{M}=\frac{1}{i}\left(\frac{\partial}{\partial \varphi_{\mathrm{p}}}+\frac{\partial}{\partial \varphi_{\mathrm{n}}}\right)
$$

The operator $\hat{P}_{M}$ eliminates the components with $m_{\mathrm{p}}+m_{\mathrm{n}} \neq$ $M$ :

$$
\hat{P}_{M} \psi\left(\varphi_{\mathrm{p}}, \varphi_{\mathrm{n}}\right)=\sum_{m_{\mathrm{p}}=-\infty}^{\infty} \sum_{m_{\mathrm{n}}=-\infty}^{\infty} \delta_{m_{\mathrm{p}}+m_{\mathrm{n}}, M} f_{m_{\mathrm{p}}} f_{m_{\mathrm{n}}} e^{i m_{\mathrm{p}} \varphi_{\mathrm{p}}} e^{i m_{\mathrm{n}} \varphi_{\mathrm{n}}} .
$$

By applying a linear transformation of the variables,

$$
\Phi=\frac{1}{2}\left(\varphi_{\mathrm{p}}+\varphi_{\mathrm{n}}\right), \quad \phi=\varphi_{\mathrm{p}}-\varphi_{\mathrm{n}},
$$

one can decouple the motions between the total rotation and the relative motion:

$$
\hat{P}_{M} \psi\left(\varphi_{\mathrm{p}}, \varphi_{\mathrm{n}}\right)=e^{i M \Phi} \sum_{\mu} f_{\frac{1}{2} M+\mu} f_{\frac{1}{2} M-\mu} e^{i \mu \phi},
$$


where

$$
M=m_{\mathrm{p}}+m_{\mathrm{n}}, \quad \mu=\frac{1}{2}\left(m_{\mathrm{p}}-m_{\mathrm{n}}\right)
$$

denote, respectively, the eigenvalues of $i^{-1} \partial / \partial \Phi$ and $i^{-1} \partial / \partial \phi$, being the momentum operators conjugate to angles $\Phi$ and $\phi$. When $M$ is an even (odd) integer, $\mu$ runs through integers (half-integers).

If an observable $\mathcal{O}$ is a function of $m_{\mathrm{p}}$ and $m_{\mathrm{n}}$ (or $M$ and $\mu$ ), its expectation value in a state projected onto angular momentum $M$ is expressed as

$$
\begin{aligned}
\langle\mathcal{O}\rangle_{M} & =\frac{\left\langle\hat{P}_{M} \psi|\mathcal{O}| \hat{P}_{M} \psi\right\rangle}{\left\langle\hat{P}_{M} \psi \mid \hat{P}_{M} \psi\right\rangle} \\
& =\frac{\sum_{m_{\mathrm{p}}}\left|f_{m_{\mathrm{p}}} f_{M-m_{\mathrm{p}}}\right|^{2} \mathcal{O}\left(m_{\mathrm{p}}, m_{\mathrm{n}}=M-m_{\mathrm{p}}\right)}{\sum_{m_{\mathrm{p}}}\left|f_{m_{\mathrm{p}}} f_{M-m_{\mathrm{p}}}\right|^{2}}
\end{aligned}
$$

or

$$
\langle\mathcal{O}\rangle_{M}=\frac{\sum_{\mu}\left|f_{\frac{1}{2} M+\mu} f_{\frac{1}{2} M-\mu}\right|^{2} \mathcal{O}(M, \mu)}{\sum_{\mu}\left|f_{\frac{1}{2} M+\mu} f_{\frac{1}{2} M-\mu}\right|^{2}}
$$

Using these equations one obtains

$$
\begin{aligned}
\left\langle m_{\mathrm{p}}^{2}\right\rangle_{M} & =\left\langle m_{\mathrm{n}}^{2}\right\rangle_{M}=\left(\frac{1}{2} M\right)^{2}+\left\langle\mu^{2}\right\rangle_{M}, \\
\left\langle m_{\mathrm{p}} m_{\mathrm{n}}\right\rangle_{M} & =\left(\frac{1}{2} M\right)^{2}-\left\langle\mu^{2}\right\rangle_{M} .
\end{aligned}
$$

For $v=2, f_{\frac{1}{2} M+\mu} f_{\frac{1}{2} M-\mu}$ on the right-hand side of Eq. (23) is proportional to $\exp \left(-a^{2} M^{2} / 2-2 a^{2} \mu^{2}\right)$ so that $\left\langle\mu^{2}\right\rangle_{M}$ becomes $\left(1 / 2^{3 / 2} a\right)^{2}$, a constant independent of $M$. In this case, the right-hand side of Eq. (28) when plotted versus $M$ expresses a parabola with a negative minimum at $M=$ 0 . Similar curves are obtained for $v>1$ from numerical calculations. These curves resemble those in Fig. 1 of Ref. [2].

As a different way of presentation, we plot the values of $\left\langle m_{\mathrm{p}} m_{\mathrm{n}}\right\rangle_{M} /\left\langle m_{\mathrm{p}}^{2}\right\rangle_{M}$ versus $M$ for $v=1.5,2$, and 3 in Fig. 2 . At $M=0$, the value is -1 , indicating that $m_{\mathrm{p}}$ and $m_{\mathrm{n}}$ have opposite signs. It increases with increasing $M$ and converges to 1 , which means that $m_{\mathrm{p}}$ and $m_{\mathrm{n}}$ have the same sign, at large $M$. This trend holds good for $v>1$ and is in agreement with the way in which two vectors $\boldsymbol{J}_{\pi}$ and $\boldsymbol{J}_{v}$ gradually align as $\boldsymbol{J}$ increases in Fig. 3 of Ref. [2]. (Numerical calculations indicate that this quantity converges to $\frac{1}{2}$ for $v=1$ and zero for $v<1$ in the limit of large $M$.) Therefore, provided $v>1$, which is satisfied by the most reasonable value of $v=2$, our model seems to include the essential ingredients to analyze the results of Ref. [2].

Does this oppositeness of the signs of $m_{\mathrm{p}}$ and $m_{\mathrm{n}}$ at small $M$ mean a free contra-rotation? With our simple model, we now show that it does not necessarily mean such a situation. If one approves the picture of the free contra-rotation, one expects a large fluctuation in the scissors mode. The size of this fluctuation can be measured by $\left\langle\phi^{2}\right\rangle_{M}$.

Some caution is necessary about $\left\langle\phi^{2}\right\rangle_{M}$. When an observable $\mathcal{O}$ is a function of $\varphi_{\mathrm{p}}$ and $\varphi_{\mathrm{n}}$, its expectation value in state



FIG. 2. The correlation between the signs of proton and neutron angular momenta. The values of $\left\langle m_{\mathrm{p}} m_{\mathrm{n}}\right\rangle_{M} /\left\langle m_{\mathrm{p}}^{2}\right\rangle_{M}$ are plotted with four types of symbols vs the total angular momentum $M$. Different symbols correspond to different angular momentum distributions $f_{m} \propto \exp \left(-|a m|^{v}\right)$ in the wave function before projection. The result for a finite number of components $(|m| \leqslant 15)$ is also displayed for $v=2$. The correspondence is indicated in the figure. The symbols are connected by lines to guide the eyes.

$\hat{P}_{M} \psi$ is expressed as

$$
\langle\mathcal{O}\rangle_{M}=\frac{\int_{-\pi}^{\pi} d \varphi_{\mathrm{p}} \int_{-\pi}^{\pi} d \varphi_{\mathrm{n}}\left|\hat{P}_{M} \psi\left(\varphi_{\mathrm{p}}, \varphi_{\mathrm{n}}\right)\right|^{2} \mathcal{O}\left(\varphi_{\mathrm{p}}, \varphi_{\mathrm{n}}\right)}{\int_{-\pi}^{\pi} d \varphi_{\mathrm{p}} \int_{-\pi}^{\pi} d \varphi_{\mathrm{n}}\left|\hat{P}_{M} \psi\left(\varphi_{\mathrm{p}}, \varphi_{\mathrm{n}}\right)\right|^{2}}
$$

However, one should not use Eq. (29) for $\mathcal{O}=\phi^{2}$ because the domain of $\phi$ corresponding to $-\pi \leqslant \varphi_{\mathrm{p}, \mathrm{n}} \leqslant \pi$ is $-2 \pi \leqslant$ $\phi \leqslant 2 \pi$. Since the relative location of the two rotors is exactly the same between $\phi$ and $\phi \pm 2 \pi$, the variable $\phi$ does not seem adequate to measure the difference of the directions between them. Instead, we choose to replace $\phi$ in the expression $\left\langle\phi^{2}\right\rangle_{M}$ by $\phi^{\prime}=\varphi_{\mathrm{p}}-\varphi_{\mathrm{n}}+2 n \pi$, where $n$ is an integer chosen such that $-\pi \leqslant \phi^{\prime}<\pi$. This replacement of $\phi$ with $\phi^{\prime}$ is equivalent to a change of the domain to $-\pi \leqslant$ $\Phi \leqslant \pi$ and $-\pi \leqslant \phi \leqslant \pi$, with which the expectation value is redefined as

$$
\langle\mathcal{O}\rangle_{M}=\frac{\int_{-\pi}^{\pi} d \Phi \int_{-\pi}^{\pi} d \phi\left|\hat{P}_{M} \psi\right|^{2} \mathcal{O}(\Phi, \phi)}{\int_{-\pi}^{\pi} d \Phi \int_{-\pi}^{\pi} d \phi\left|\hat{P}_{M} \psi\right|^{2}},
$$

for which the replacement of $\phi$ with $\phi^{\prime}$ is not necessary. In numerical calculations, we have used Eq. (30) because Eq. (29) makes integrals less precise because of the discontinuity of the derivative of $\phi^{\prime}$ inside the integral domain.

For $v=2$, one can show, using $a \ll \pi$,

$$
\hat{P}_{M} \psi\left(\varphi_{\mathrm{p}}, \varphi_{\mathrm{n}}\right)=\frac{1}{2 \pi} e^{-a^{2} M^{2} / 2} e^{i M \Phi} e^{-\left(\phi / 2^{3 / 2} a\right)^{2}},
$$




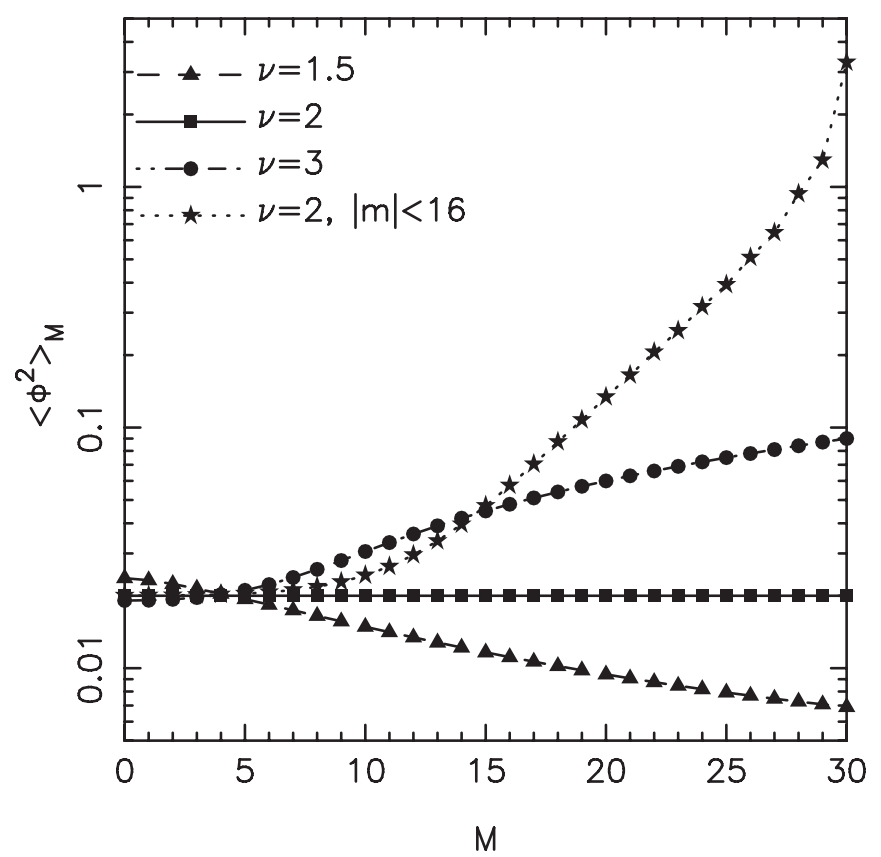

FIG. 3. The fluctuation in the scissors mode $\left\langle\phi^{2}\right\rangle_{M}$ vs the total angular momentum $M$. It is independent of $M$ for $v=2$. For $v>2(v<2)$, it increases (decreases) with increasing $M$. With a finite number of components $(|m| \leqslant 15)$, the band termination occurs at $M=30$, where $\left\langle\phi^{2}\right\rangle_{M}$ takes on the uncorrelated-rotation value $\frac{1}{3} \pi^{2}$.

which indicates $\left\langle\phi^{2}\right\rangle_{M}=2 a^{2}$. It is much smaller than $\frac{1}{3} \pi^{2}$, which is expected for free contra-rotation or uncorrelated rotations. In Fig. 3, $\left\langle\phi^{2}\right\rangle_{M}$ is plotted for $v=1.5,2$, and 3. In every case, the fluctuation in $\phi$ is $\sim a$ at small $M$ where $\left\langle m_{\mathrm{p}} m_{\mathrm{n}}\right\rangle_{M}$ is negative. Even by increasing $v$ to infinity, we observe that $\left\langle\phi^{2}\right\rangle_{M}$ remains less than 0.1 for $M \leqslant$ 7. This fact is incompatible with the free contra-rotation picture. One can also see by comparing Fig. 2 and Fig. 3 that $\left\langle\phi^{2}\right\rangle_{M}$ is much more sensitive than $\left\langle m_{\mathrm{p}} m_{\mathrm{n}}\right\rangle_{M}$ to the angular momentum distribution of the wave function before projection.

When the number of angular momentum components is finite $\left(\left|m_{\mathrm{p}, \mathrm{n}}\right| \leqslant 15\right)$, the fluctuation can become very large at high spins, reaching $\frac{1}{3} \pi^{2}$ at the maximum possible angular momentum $M=30$ (see Fig. 3). This enhancement at high spins is associated with the band termination phenomenon [16], i.e., with free rotations in the same direction, not with the free contra-rotation.

In Fig. $4,\left\langle\mu^{2}\right\rangle_{M}$ is plotted for $v=1.5,2$, and 3. By comparing this figure with Fig. 3, one can confirm the complementarity between $\hat{\mu}=i^{-1} \partial / \partial \phi$ and $\phi$ imposed by the uncertainty principle.

Another way to see clearly how the angular momentum projection produces such strongly correlated states out of the completely uncorrelated state $f_{\mathrm{p}}\left(\varphi_{\mathrm{p}}\right) f_{\mathrm{n}}\left(\varphi_{\mathrm{n}}\right)$ is to express the projection as an integral over the angle $\Phi$ [17]. By a substitution

$$
\delta_{m_{\mathrm{p}}+m_{\mathrm{n}}, M}=\frac{1}{2 \pi} \int_{-\pi}^{\pi} e^{i\left(M-m_{\mathrm{p}}-m_{\mathrm{n}}\right) \Phi} d \Phi,
$$

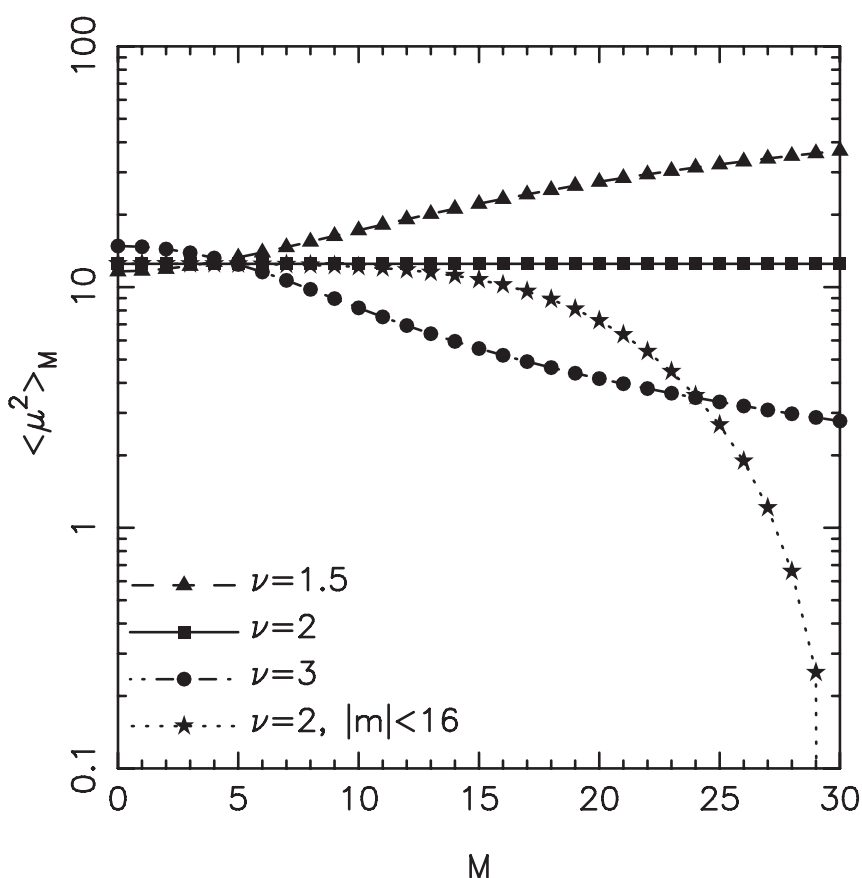

FIG. 4. The mean square value of the relative angular momentum $\left\langle\mu^{2}\right\rangle_{M}$ vs the total angular momentum $M$. For $v=2$ it is a constant, while for $v>2(v<2)$ it decreases (increases) with increasing $M$. With a finite number of components $(|m| \leqslant 15)$, it becomes zero at the band termination $(M=30)$.

one can rewrite Eq. (21) as

$\hat{P}_{M} \psi=\frac{1}{2 \pi} \int_{-\pi}^{\pi} e^{i M \Phi} \sum_{m_{\mathrm{p}}} \sum_{m_{\mathrm{n}}} f_{m_{\mathrm{p}}} f_{m_{\mathrm{n}}} e^{i m_{\mathrm{p}}\left(\varphi_{\mathrm{p}}-\Phi\right)} e^{i m_{\mathrm{n}}\left(\varphi_{\mathrm{n}}-\Phi\right)} d \Phi$,

and using Eqs. (16) and (17),

$\hat{P}_{M} f_{\mathrm{p}}\left(\varphi_{\mathrm{p}}\right) f_{\mathrm{n}}\left(\varphi_{\mathrm{n}}\right)=\frac{1}{2 \pi} \int_{-\pi}^{\pi} e^{i M \Phi} f_{\mathrm{p}}\left(\varphi_{\mathrm{p}}-\Phi\right) f_{\mathrm{n}}\left(\varphi_{\mathrm{n}}-\Phi\right) d \Phi$.

Equation (34) means that the projected state is a superposition of states obtained by rotating the two rotors by the same angle, i.e., moving the two rotors together.

From the discussions given in this section, we conclude that the fact that the angular momentum of protons and that of neutrons are oriented in opposite directions does not indicate that the ellipsoids of the protons and the neutrons are freely rotating in the opposite sense. On the contrary, it is a consequence of the close binding of the protons and the neutrons: At small angular momentum $M$, the fluctuation in the scissors mode $\left\langle\phi^{2}\right\rangle_{M}$ is as small as in the wave function before the projection. To achieve this, however, the uncertainty principle imposes a large spreading of the conjugate angular momentum $\mu=\frac{1}{2}\left(m_{\mathrm{p}}-m_{\mathrm{n}}\right)$. When $M=m_{\mathrm{p}}+m_{\mathrm{n}}$ is much smaller than $\left\langle\mu^{2}\right\rangle_{M}^{1 / 2}$, it inevitably holds $m_{\mathrm{p}} \sim-m_{\mathrm{n}}$.

In other words, the picture of a free contra-rotation may be appropriate for a wave function consisting of a single component $\exp \left(i m \varphi_{\mathrm{p}}-i m \varphi_{\mathrm{n}}\right)$. By adding up many components satisfying $m_{\mathrm{p}}+m_{\mathrm{n}}=M$, however, one obtains 
a very different wave function in which protons and neutrons are bound together closely rather than rotating freely in the opposite sense.

In this paper, we have introduced a wave function without specifying the underlying Hamiltonian, giving a new and more precise insight of nuclear rotation at low rotational frequency. The dynamical aspect regarding the $M 1$ sum rule discussed briefly in Ref. [4] can be nicely implemented into the present two-dimensional model as reported elsewhere.

A way to advance further is to allow the ellipsoids to rotate three-dimensionally. In this case, the angular momentum vectors of the two ellipsoids can be at angles other than $0^{\circ}$ or $180^{\circ}$, where the latter corresponds to the ground state. As the angle decreases gradually from $180^{\circ}$, spatial overlap between the wave function of the proton ellipsoid and that of neutron ellipsoid becomes smaller, resulting in less energy gain from the attractive proton-neutron interaction. Reference [2] presented rather extensive discussions on this mechanism, but not on the contra-rotation picture. It is of interest to examine further to what extent such a mechanism plays an important role in generating rotational spectra.

\section{ACKNOWLEDGMENT}

The authors are grateful to Professors A. Richter and P. von Neumann-Cosel for valuable comments.

\section{APPENDIX}

In this Appendix we estimate the root-mean-square values of $\boldsymbol{L}_{\tau}, \tilde{\boldsymbol{L}}_{\tau}$, and $\boldsymbol{\Sigma}_{\tau}$ in classical mechanics.

Our estimation is for the state before the angular momentum projection, in which nucleons are moving in a space-fixed deformed potential like the Nilsson potential. We assume that the nucleons move independently of one another and that the momentum distribution is locally isotropic. Namely, the distribution function in the $N$-body phase space is given by

$$
F\left(\boldsymbol{r}_{1}, \ldots, \boldsymbol{r}_{N}, \boldsymbol{p}_{1}, \ldots, \boldsymbol{p}_{N}\right)=\prod_{i} F_{\mathrm{sp}}\left(\boldsymbol{r}_{i},\left|\boldsymbol{p}_{i}\right|\right),
$$

with which the expectation value of an observable $Q\left(\boldsymbol{r}_{1}, \ldots, \boldsymbol{r}_{N}, \boldsymbol{p}_{1}, \ldots, \boldsymbol{p}_{N}\right)$ is calculated as

$$
\langle Q\rangle=\int F\left(\boldsymbol{r}_{1}, \ldots, \boldsymbol{p}_{N}\right) Q\left(\boldsymbol{r}_{1}, \ldots, \boldsymbol{p}_{N}\right) \prod_{i} d^{3} r_{i} d^{3} p_{i}
$$

Using different $F_{\mathrm{sp}}$ between protons and neutrons would not bring about essential difficulties to the following estimations.

The function $F$ affects the final results only through three single-particle expectation values,

$$
\begin{aligned}
\bar{r}^{2} & =\left\langle\boldsymbol{r}^{2}\right\rangle_{\mathrm{sp}}, \\
\bar{p}^{2} & =\left\langle\boldsymbol{p}^{2}\right\rangle_{\mathrm{sp}}, \\
\alpha & =\frac{\left\langle\boldsymbol{r}^{2} \boldsymbol{p}^{2}\right\rangle_{\mathrm{sp}}}{\bar{r}^{2} \bar{p}^{2}}-1,
\end{aligned}
$$

where

$$
\langle Q\rangle_{\mathrm{sp}}=\int F_{\mathrm{sp}}(\boldsymbol{r},|\boldsymbol{p}|) Q(\boldsymbol{r}, \boldsymbol{p}) d^{3} r d^{3} p .
$$

The value of $\alpha$ is probably much smaller than one because it becomes zero if $F_{\mathrm{sp}}(\boldsymbol{r},|\boldsymbol{p}|)$ can be factorized into a form $F_{1}(\boldsymbol{r}) F_{2}(|\boldsymbol{p}|)$, which is true in the Thomas-Fermi approximation for a potential having a flat bottom and a sharp surface, i.e., a very simplified nuclear potential.

Our aim is to evaluate the expectation values of three observables,

$$
\begin{aligned}
\left\langle\boldsymbol{L}_{\tau}^{2}\right\rangle & =\frac{1}{N_{\tau}^{2}} \sum_{i j k l \in \tau}\left\langle\left(\boldsymbol{r}_{i} \times \boldsymbol{p}_{j}\right) \cdot\left(\boldsymbol{r}_{k} \times \boldsymbol{p}_{l}\right)\right\rangle, \\
\left\langle\tilde{\boldsymbol{L}}_{\tau}^{2}\right\rangle & =\frac{1}{N_{\tau}^{2}} \sum_{i j k l \in \tau}\left\langle\left(\tilde{\boldsymbol{r}}_{i} \times \tilde{\boldsymbol{p}}_{j}\right) \cdot\left(\tilde{\boldsymbol{r}}_{k} \times \tilde{\boldsymbol{p}}_{l}\right)\right\rangle, \\
\left\langle\boldsymbol{\Sigma}_{\tau}^{2}\right\rangle & =\left\langle\boldsymbol{l}_{\tau}^{2}-2 \boldsymbol{l}_{\tau} \cdot \boldsymbol{L}_{\tau}+\boldsymbol{L}_{\tau}^{2}\right\rangle .
\end{aligned}
$$

The last equation requires the evaluation of two additional quantities,

$$
\begin{aligned}
\left\langle\boldsymbol{l}_{\tau}^{2}\right\rangle & =\sum_{i j \in \tau}\left\langle\left(\boldsymbol{r}_{i} \times \boldsymbol{p}_{i}\right) \cdot\left(\boldsymbol{r}_{j} \times \boldsymbol{p}_{j}\right)\right\rangle, \\
\left\langle\boldsymbol{l}_{\tau} \cdot \boldsymbol{L}_{\tau}\right\rangle & =\frac{1}{N_{\tau}} \sum_{i j k \in \tau}\left\langle\left(\boldsymbol{r}_{i} \times \boldsymbol{p}_{i}\right) \cdot\left(\boldsymbol{r}_{j} \times \boldsymbol{p}_{k}\right)\right\rangle .
\end{aligned}
$$

Equations (A7), (A10), and (A11) include terms of the form

$$
\begin{aligned}
\left\langle\left(\boldsymbol{r}_{i} \times \boldsymbol{p}_{j}\right) \cdot\left(\boldsymbol{r}_{k} \times \boldsymbol{p}_{l}\right)\right\rangle= & \left\langle\left(\boldsymbol{r}_{i} \cdot \boldsymbol{r}_{k}\right)\left(\boldsymbol{p}_{j} \cdot \boldsymbol{p}_{l}\right)\right\rangle \\
& -\left\langle\left(\boldsymbol{r}_{i} \cdot \boldsymbol{p}_{l}\right)\left(\boldsymbol{r}_{k} \cdot \boldsymbol{p}_{j}\right)\right\rangle .
\end{aligned}
$$

The two terms on the right-hand side of Eq. (A12) can be calculated as phase-space integrals according to Eqs. (A1) and (A2). By noting

$$
\begin{gathered}
\int F\left(\boldsymbol{r}_{i},\left|\boldsymbol{p}_{i}\right|\right) p_{i \kappa} d^{3} p_{i}=0 \\
\int F\left(\boldsymbol{r}_{i},\left|\boldsymbol{p}_{i}\right|\right) p_{i \mu} p_{i \nu} d^{3} p_{i} \propto \delta_{\mu \nu}
\end{gathered}
$$

where $\kappa, \mu, v=x, y, z$ and $\boldsymbol{p}_{i}=\left(p_{i x}, p_{i y}, p_{i z}\right)$, one can obtain

$$
\begin{aligned}
& \left\langle\left(\boldsymbol{r}_{i} \cdot \boldsymbol{r}_{j}\right)\left(\boldsymbol{p}_{k} \cdot \boldsymbol{p}_{l}\right)\right\rangle=\delta_{i j} \delta_{k l}\left(1+\alpha \delta_{i k}\right) \bar{r}^{2} \bar{p}^{2}, \\
& \left\langle\left(\boldsymbol{r}_{i} \cdot \boldsymbol{p}_{j}\right)\left(\boldsymbol{r}_{k} \cdot \boldsymbol{p}_{l}\right)\right\rangle=\frac{1}{3} \delta_{i k} \delta_{j l}\left(1+\alpha \delta_{i j}\right) \bar{r}^{2} \bar{p}^{2},
\end{aligned}
$$

and thus

$$
\left\langle\left(\boldsymbol{r}_{i} \times \boldsymbol{p}_{j}\right) \cdot\left(\boldsymbol{r}_{k} \times \boldsymbol{p}_{l}\right)\right\rangle=\frac{2}{3} \delta_{i k} \delta_{j l}\left(1+\alpha \delta_{i j}\right) \bar{r}^{2} \bar{p}^{2} .
$$

Using Eq. (A17), one obtains

$$
\begin{aligned}
\left\langle\boldsymbol{L}_{\tau}^{2}\right\rangle & =\frac{2}{3}\left(1+\frac{\alpha}{N_{\tau}}\right) \bar{r}^{2} \bar{p}^{2}, \\
\left\langle\boldsymbol{l}_{\tau}^{2}\right\rangle & =\frac{2}{3} N_{\tau}(1+\alpha) \bar{r}^{2} \bar{p}^{2}, \\
\left\langle\boldsymbol{l}_{\tau} \cdot \boldsymbol{L}_{\tau}\right\rangle & =\frac{2}{3}(1+\alpha) \bar{r}^{2} \bar{p}^{2} .
\end{aligned}
$$


Substituting terms in Eq. (A9) with Eqs. (A18)-(A20), one finds

$$
\left\langle\Sigma_{\tau}^{2}\right\rangle=\frac{2}{3}\left[N_{\tau}-1+\alpha N_{\tau}\left(1-\frac{1}{N_{\tau}}\right)^{2}\right] \bar{r}^{2} \bar{p}^{2} .
$$

Equation (A8) includes a term of the form

$$
\begin{aligned}
\left\langle\left(\tilde{\boldsymbol{r}}_{i} \times \tilde{\boldsymbol{p}}_{j}\right) \cdot\left(\tilde{\boldsymbol{r}}_{k} \times \tilde{\boldsymbol{p}}_{l}\right)\right\rangle= & \left\langle\left(\tilde{\boldsymbol{r}}_{i} \cdot \tilde{\boldsymbol{r}}_{k}\right)\left(\tilde{\boldsymbol{p}}_{j} \cdot \tilde{\boldsymbol{p}}_{l}\right)\right\rangle \\
& -\left\langle\left(\tilde{\boldsymbol{r}}_{i} \cdot \tilde{\boldsymbol{p}}_{l}\right)\left(\tilde{\boldsymbol{r}}_{k} \cdot \tilde{\boldsymbol{p}}_{j}\right)\right\rangle .
\end{aligned}
$$

Terms like the first one on the right-hand side of Eq. (A22) can be calculated using Eq. (A15) as

$$
\begin{aligned}
\left\langle\left(\tilde{\boldsymbol{r}}_{i} \cdot \tilde{\boldsymbol{r}}_{j}\right)\left(\tilde{\boldsymbol{p}}_{k} \cdot \tilde{\boldsymbol{p}}_{l}\right)\right\rangle & \left\langle\left(\boldsymbol{r}_{i}-\frac{1}{N} \sum_{i^{\prime}} \boldsymbol{r}_{i^{\prime}}\right) \cdot\left(\boldsymbol{r}_{j}-\frac{1}{N} \sum_{j^{\prime}} \boldsymbol{r}_{j^{\prime}}\right)\right. \\
& \left.\times\left(\boldsymbol{p}_{k}-\frac{1}{N} \sum_{k^{\prime}} \boldsymbol{p}_{k^{\prime}}\right) \cdot\left(\boldsymbol{p}_{l}-\frac{1}{N} \sum_{l^{\prime}} \boldsymbol{p}_{l^{\prime}}\right)\right\rangle \\
= & \left\langle\left(\boldsymbol{r}_{i} \cdot \boldsymbol{r}_{j}\right)\left(\boldsymbol{p}_{k} \cdot \boldsymbol{p}_{l}\right)\right\rangle-\frac{1}{N} \sum_{i^{\prime}}\left\langle\left(\boldsymbol{r}_{i^{\prime}} \cdot \boldsymbol{r}_{j}\right)\left(\boldsymbol{p}_{k} \cdot \boldsymbol{p}_{l}\right)\right\rangle \\
& +(14 \text { terms })
\end{aligned}
$$

$$
\begin{aligned}
= & \delta_{i j} \delta_{k l}\left(1+\alpha \delta_{i k}\right) \bar{r}^{2} \bar{p}^{2}-\frac{1}{N} \delta_{k l}\left(1+\alpha \delta_{j k}\right) \bar{r}^{2} \bar{p}^{2} \\
& +(14 \text { terms }) .
\end{aligned}
$$

A similar 16-term expression can be obtained for terms like $\left\langle\left(\tilde{\boldsymbol{r}}_{i} \cdot \tilde{\boldsymbol{p}}_{j}\right)\left(\tilde{\boldsymbol{r}}_{k} \cdot \tilde{\boldsymbol{p}}_{l}\right)\right\rangle$. Using such expressions, one obtains

$$
\begin{aligned}
\left\langle\tilde{\boldsymbol{L}}_{\tau}^{2}\right\rangle= & \frac{2}{3}\left(1-\frac{N_{\tau}}{N}\right) \\
& \times\left[1-\frac{N_{\tau}}{N}+\frac{\alpha}{N_{\tau}}\left(1-3 \frac{N_{\tau}}{N}+3 \frac{N_{\tau}^{2}}{N^{2}}\right)\right] \bar{r}^{2} \bar{p}^{2} .
\end{aligned}
$$

Because $1 \ll N_{\tau}<N$ for typical rotational nuclei, one can conclude $\left\langle\tilde{\boldsymbol{L}}_{\tau}^{2}\right\rangle \sim\left\langle\boldsymbol{L}_{\tau}^{2}\right\rangle \ll\left\langle\boldsymbol{\Sigma}_{\tau}^{2}\right\rangle$.

What we have shown above is not an estimation for the state after the projection to an angular momentum of zero, which requires a much more difficult task of restricting the $N$-particle phase space to a manifold defined by $\boldsymbol{l}=0$ or $\tilde{\boldsymbol{l}}=0$. However, we do not think that it would change the conclusion substantially because low angular momentum components have large probabilities in the state before projection.
[1] A. Bohr and B. R. Mottelson, Nuclear Structure (Benjamin, New York, 1975), Vol. 2.

[2] T. Otsuka, Phys. Rev. Lett. 71, 1804 (1993).

[3] T. Otsuka, T. Mizusaki, and M. Honma, Nucl. Phys. A 570, 265c (1994).

[4] T. Otsuka and J. N. Ginocchio, Nucl. Phys. A 577, 197c (1994).

[5] N. Lo Iudice and F. Palumbo, Phys. Rev. Lett. 41, 1532 (1978).

[6] D. Bohle, A. Richter, W. Steffen, A. E. L. Dieperink, N. Lo Iudice, F. Palumbo, and O. Scholten, Phys. Lett. B 137, 27 (1984).

[7] A. S. Adekola, C. T. Angell, S. L. Hammond, A. Hill, C. R. Howell, H. J. Karwowski, J. H. Kelley, and E. Kwan, Phys. Rev. C 83, 034615 (2011).

[8] K. Heyde, P. von Neumann-Cosel, and A. Richter, Rev. Mod. Phys. 82, 2365 (2010).

[9] W. Bentz, A. Arima, J. Enders, A. Richter, and J. Wambach, Phys. Rev. C 84, 014327 (2011).
[10] K. Hatada, K. Hayakawa, and F. Palumbo, Phys. Rev. C 84, 011302 (2011).

[11] E. R. Marshalek, Phys. Rev. C 50, R5 (1994).

[12] Y. Sun, C.-L. Wu, K. Bhatt, M. Guidry, and D. H. Feng, Phys. Rev. Lett. 80, 672 (1998).

[13] D. R. Bes and O. Civitarese, Phys. Rev. C 63, 044323 (2001).

[14] W. Ziegler, N. Huxel, P. von Neumann-Cosel, C. Rangacharyulu, A. Richter, C. Spieler, C. De Coster, and K. Heyde, Nucl. Phys. A 564, 366 (1993).

[15] W. Ziegler, C. Rangacharyulu, A. Richter, and C. Spieler, Phys. Rev. Lett. 65, 2515 (1990).

[16] S. G. Nilsson and I. Ragnarsson, Shapes and Shells in Nuclear Structure (Cambridge University Press, Cambridge, 1995).

[17] P. Ring and P. Schuck, The Nuclear Many-Body Problem (Springer, New York, 1980). 